\title{
The Theory of Static Gravitational Field
}

\author{
Jaroslav Hynecek ${ }^{1}$ \\ ${ }^{1}$ Isetex Inc., 905 Pampa Drive, Allen, TX, USA \\ Correspondence: Jaroslav Hynecek, Isetex Inc., 905 Pampa Drive, Allen, TX 75013, USA. E-mail: \\ jhynecek@netscape.net
}

Received: July 23, 2012 Accepted: August 16, 2012 Online Published: October 12, 2012

doi:10.5539/apr.v4n4p44

URL: http://dx.doi.org/10.5539/apr.v4n4p44

\begin{abstract}
The paper discusses in detail assumptions needed for the derivation of metric for the non-rotating centrally gravitating body, which is not based on Einstein field equations. The metric derivation is then generalized to any static mass configuration. It is shown that the Schwarzschild metric, which is the famous solution of Einstein field equations for the centrally gravitating body, is only a first order approximation of the correct metric and that the Einstein field equation concept is a wrong concept for finding any metric. Several examples of observations in support of the developed theory, which are not as easily explained in the main stream literature, are also presented in this paper.
\end{abstract}

Keywords: Minkowski space-time, general relativity theory, Einstein field equations, black holes, Schwarzschild metric, Ricci scalar, correct metric for the centrally gravitating body, aether, dark matter

\section{Introduction}

Any modern theory that has been constructed so far is based on assumptions that lead to conclusions that are more or less in agreement with observations or are somehow "self evident". This does not mean that the theory is absolutely true or that it cannot be later corrected, modified, improved, or proven wrong when more precise data become available for comparison. However, the theory must be clear in defining these assumptions and the assumptions cannot contradict each other or the well established laws of physics. The well known procedure to work these problems out is to use a suitable well proven mathematical formalism, so the math is a valuable tool to resolve such conflicts should they arise. However, the mathematical formalism must be clear and not self-absorbing with many abstract notions that the mathematicians like so much to the point that the physics becomes completely lost in it. An example of this problem is the variational principle for the derivation of Einstein field equations followed by the arbitrary assumption about the mass energy tensor. The theory that will be derived in this paper will avoid these pitfalls.

However, before we embark on the derivation of new metric it is also necessary to make few comments about the space-time and coordinates.

\section{Space-time}

There are many misconception in the scientific world today about the space and time. This leads to a confusion and many wrong and imprecise ideas including the various space-time theories. The main reason for this confusion is that the mathematicians have abstracted the notion of coordinates form the actual reality and use them in their abstract mathematical theories such as, for example, in tensor calculus of General Relativity Theory (GRT). This seemingly gives them an independent real existence. However, this is not correct, the coordinates by themselves are just an abstract mathematical tool without the real physical meaning. The coordinates should always be connected to some real entities, which may, for example, be called the space-time. The coordinates by themselves are not the space-time.

This can be compared to numbers. The numbers by themselves do not have any physical meaning until we connect them to some real objects. This is the fundamental difference between the physics and the mathematics, which we must always keep in mind. The real physical objects must also have some other physical properties in addition to just being able to be counted. They must have the size, the mass, the temperature, the stiffness, the dielectric constant, the index of refraction, etc. The same is true for the coordinates.

The space that the coordinates describe must also have some other physical properties in addition to its size and 
distances if it should have a real physical existence. The notion that there is a vacuum between objects with nothing in it cannot be correct. The description of the space-time as is proposed by the GRT with nothing in the space between the massive objects just an "empty space" around these objects cannot be correct. The space around the central mass must have some other physical properties in addition to distances. There is a field in this space, the space allows propagation of light, so it must have some elasticity and the mass density, it must have a temperature and perhaps also an internal pressure etc.

Since the space allows the propagation of disturbances in form of waves with a certain velocity, this automatically defines time. So, when we speak about the space-time we must consider that it is a physical entity with perhaps a very small but real mass density, the stiffness, the temperature, the ability to support the wave propagation etc., not only that it has abstract distances. It is therefore clear that the space-time must be some sort of physical medium, perhaps as it was called in the past the aether, or as it is called today the dark matter, which supports all the remaining physical properties that the GRT has conveniently dropped from considerations.

The coordinates by themselves are not the space-time, they only describe one aspect of it.

\section{Potential Derivation}

Before embarking on this topic, it is necessary to mention the coordinates once more. In this paper there will be two kinds of coordinates used. The natural coordinates, sometimes called just coordinates, these are the ones that we are using for measurement of distances and time by sticks and clocks; and the physical coordinates that will be used in physics formulas. The reason for this is the key assumption of this theory that the natural coordinates, the space-time we are living in, are distorted by gravity. The physical coordinates, on the other hand, will describe an abstract undistorted flat space-time into which the natural space-time is mapped. The physical coordinates are thus not directly observable or measurable, they are absolute, unique, and must be calculated from the natural coordinates using some derived physics formulas. This is why these coordinates have been named physical.

The metric derivation for the centrally gravitating non-rotating body will begin with the definition of potential. It is our experience and observation that there is a gravitational force that attracts all masses. Barring the loss of energy by the gravitational wave radiation, or various frictional losses, it is reasonable to assume that the static gravitational force can be represented by a field that possesses a potential. This also means that the path integral calculating the work of a small test body along a closed path in this field is zero. This, for example, implies that the orbits of planets (viewed as small test bodies) around the Sun are stable and do not significantly decay over the period of many years as we are all well aware of. The potential in such a centrally gravitating system where the trajectories are conserving energy thus has a corresponding field that can be calculated by minimizing the energy stored in this field according to the following variational principle:

$$
\delta \int_{R}\left[\left(\frac{\partial \varphi_{n}}{\partial \rho}\right)^{2}-\Lambda\left(\varphi_{n}\right)\right] \rho^{2} d \rho=0
$$

where $\rho$ is the generalized physical radius to be subsequently defined in more detail. The second term in the square parentheses represents the background energy of the space itself, but for the purposes of this paper this term will be neglected, since it will be assumed very small in the neighborhood of gravitating bodies. However, this term plays an important role on the cosmological scales when the whole universe is studied. The Euler-Lagrange (EL) equation corresponding to this simplified variational problem is thus as follows:

$$
\frac{d}{d \rho}\left(\frac{\partial \varphi_{n}}{\partial \rho} \rho^{2}\right)=0
$$

In these equations the natural radius $r$ was replaced by a physical radius $\rho=\rho(r)$ since it is necessary to include into considerations a possibility that the usual radial coordinate is distorted by the gravity while $\rho$ remains unaffected by it as already mentioned. The gravitational field will be actually considered as resulting from the deformation of the natural space-time we are living in. The observed natural coordinate distances and the natural coordinate time in the curved space-times thus do not have a direct physical meaning as in the flat space-time. The relation between the physical radius and the natural coordinate radius is expressed by using the well known metric coefficient and by the well known formula that can be written in the spherically symmetrical coordinate system as follows:

$$
d \rho=\sqrt{g_{r r}} d r
$$


The solution of Equation 2 is easily obtained using the zero boundary condition at infinity and can be written as:

$$
\varphi_{n}=-\frac{\kappa M}{\rho(r)}
$$

where $M$ is the mass of the centrally gravitation body and $\kappa$ is the gravitational constant. This is the famous Newton gravitational potential with the modification of replacing the natural coordinate radius by the physical radius that is necessary for the curved space-time. However, to proceed further it is also necessary to find the relation between the metric coefficient and the potential using some other physical reasoning since the substitution of the physical radius for the natural coordinate radius at this stage of derivation is essentially only a formality.

\section{Metric Derivation}

In the Einstein's General Relativity Theory it is assumed that the metric coefficients and not the potential are the field variables and the principle that is used to find them is to find the stationary point of the variational integral with the Lagrangian: $R_{(c)}(-g)^{-1 / 2}$, where $R_{(\mathrm{c})}$ is the Ricci scalar that depends on the second derivatives of these coefficients. The variational integral is usually written as:

$$
\delta \int_{\Sigma} R_{(c)} \sqrt{-g} d x^{4}=0
$$

with $g$ being the natural coordinate metric determinant. Solution of this variational principle are the famous Einstein field equations:

$$
G_{j k}=R_{j k}-\frac{1}{2} R_{(c)} g_{j k}=-\frac{8 \pi \kappa}{c^{4}} T_{j k}, \quad T_{k \mid j}^{j}=0
$$

While this is a widely accepted concept, believed to be so beautiful that it is beyond any reproach, it is not difficult to raise some obvious objections to it. The Ricci scalar is the consequence, the effect caused by the gravitational field, resulting from the presence of the mass that distorts the natural coordinate space-time. Therefore, minimizing the result instead of the cause, which is the field energy, does not make much sense in particular when the relation between the field energy and the Ricci scalar is not linear. Despite this obvious fact sometimes the claims are being made in the literature that the field energy is already included in the Ricci tensor, $R_{j k}$ but this cannot be correct, since this tensor is zero in places where the field is not zero. The second problem is that the mass energy tensor $T_{j k}$ is not calculated from the formalism itself and must be determined using some other physical reasoning. For the centrally gravitating body it is believed and assumed that it is zero outside of the massive body. This is a strange assumption claiming that in the space where the field is the mass energy tensor is zero. The third problem is that the variational principle in Equation 5 does not guarantee that the field energy will also be at its minimum when the above mentioned variational integral is at its stationary point. The well known and the time tested principle of minimum field energy is not used here. It has not been proven that the variational principle introduced in Equation 5 also implies the minimum field energy. Actually, due to the general covariance principle it is sometimes claimed that there is no energy in the gravitational field or that the field energy is not localized since by transforming the coordinates into a free falling coordinate system the field can be transformed away. Setting the mass energy tensor to zero is not equivalent to finding the minimum value for the field energy. Actually, the zero value for this tensor may not be permissible for the nonzero value of the gravitating mass except perhaps in the weak field limit. More details about the problems of GRT can be found in Hynecek (2009a), Hynecek (2011), and Logunov (1983).

The solution of Equation 6 for the zero mass energy tensor is the famous Schwarzschild metric:

$$
d s^{2}=g_{t t}(c d t)^{2}-g_{t t}^{-1} d r^{2}-r^{2} d \Omega^{2}
$$

where the metric coefficient $g_{t t}$ equals to:

$$
g_{t t}=1-R_{s} / r
$$

and the Schwarzschild radius $R_{s}$ is defined as:

$$
R_{s}=\frac{2 \kappa M}{c^{2}}
$$

The usual angular coordinate term: $\left(d \vartheta^{2}+\sin ^{2} \vartheta d \phi^{2}\right)$ used in the Schwarzschild metric line element in Equation 
7 was replaced here by a more general angular coordinate term $d \Omega^{2}$ in order to emphasize the coordinate orthogonality and the spherical symmetry. However, the Schwarzschild solution produces a coordinate problem at the Schwarzschild radius called the "event horizon", which then leads to the Black Hole (BH) theories and other unreasonable theoretical creations that may not exist in reality and have yet to be confirmed by observations.

It thus seems that the primary source of all these problems and objections is the unusual and nowhere else in physics encountered variational principle of Equation 5 where the result instead of the primary cause are varied thus leading to the nonphysical Einstein field equations with observational proofs of solutions obtained only for the weak gravitational fields and leading to the unreasonable event horizon artifact.

All these problems stem from the fact that there was no physical entity considered behind the natural coordinates. It was considered that the space-time is just an abstract "empty space", a vacuum. But when we ascribe some physical properties to the natural space-time, such as the mass density, however small it might be, and a large stiffness, it is immediately obvious that there must be a field energy in that space-time, which originates from the space-time distortion caused by the gravitating mass.

In the following sections the Einstein approach of finding the metric will, therefore, be abandoned and the metric for the centrally gravitating body will be found from the more fundamental principles of physics, which have been verified may times before and are without any doubts. The metric will be found by having a small test body moving in the space-time of the studied metric and it will be investigated whether the trajectories satisfy the well known principles such as the trajectory equation covariance and the conservation of angular momentum. The trajectories that will satisfy these requirements will then be considered correct, corresponding to reality, and the metric that provides them the correct space-time metric. It will also be considered that the deformation of the natural coordinate space-time by the gravity of the centrally gravitating body is locally isotropic.

The general metric line element of the centrally gravitating body taking an advantage of coordinate orthogonality and the spherical symmetry is as follows:

$$
d s^{2}=g_{t t}(c d t)^{2}-g_{r r} d r^{2}-g_{\varphi \varphi} d \Omega^{2}
$$

The angular coordinate metric coefficient will be obtained by considering for simplicity a small test body orbiting the main gravitating body only in the equatorial plane. The Lagrangian describing such a motion corresponding to the metric line element in Equation 10 is as follows:

$$
L=g_{t t}\left(\frac{c d t}{d \tau}\right)^{2}-g_{r r}\left(\frac{d r}{d \tau}\right)^{2}-g_{\varphi \varphi}\left(\frac{d \varphi}{d \tau}\right)^{2}
$$

The EL equations of motion are derived from the variational principle:

$$
\delta \int_{\tau} L d \tau=0
$$

with their first integrals easily found to be:

$$
\begin{gathered}
g_{t t} \frac{d t}{d \tau}=1 \\
g_{\varphi \varphi} \frac{d \varphi}{d \tau}=\alpha
\end{gathered}
$$

where $\alpha$ is an integration constant corresponding to the angular momentum. Eliminating the non-observable parameter $d \tau$ from these formulas leads to the well known equation for the conservation of angular momentum.

$$
\frac{g_{\varphi \varphi}}{g_{t t}} \frac{d \varphi}{d t}=\alpha
$$

From this formula it is then clear that the ratio of the angular metric coefficient and the coordinate time metric coefficient must have a dimension of a radius squared. Therefore, by maintaining the consistency with the approach to the Newton gravitational potential where the natural coordinate radius was replaced by the physical radius, the ratio of these two metric coefficients will be the physical radius squared instead of the natural coordinate radius squared. It will thus be considered that it must hold the following: 


$$
g_{\varphi \varphi}=\rho^{2} g_{t t}
$$

For the Schwarzschild metric, however, the equivalent of Equation 15 is:

$$
\frac{r^{2}}{\left(1-R_{s} / r\right)} \frac{d \varphi}{d t}=\alpha
$$

This suggests that the angular momentum as is commonly known to be conserved is not conserved here. In particular for the natural coordinate radius approaching the Schwarzschild radius the angular velocity would approach zero, so no spin interaction could be observed transferred into or out of the BH. This is again not reasonable, since the angular momentum cannot be lost from the matter forming the gravitating body when the BH is purportedly created. In addition it is well known that the very compact stars such as the neutron stars spin at very high rates. The neutron stars have their radius close to the Schwarzschild radius and thus their spin should be very slow. This, however, contradicts the observations.

For the derivation of the metric coefficients standing by the natural radial coordinate and the natural time coordinate it is useful to consider for simplicity a test body falling only in the radial direction. The Lagrangian describing such a motion is as follows:

$$
L=g_{t t}\left(\frac{c d t}{d \tau}\right)^{2}-g_{r r}\left(\frac{d r}{d \tau}\right)^{2}
$$

The EL equations of motion obtained from the variational principle shown in Equation 12 are as follows:

$$
\begin{gathered}
\frac{d}{d \tau}\left(g_{t t} \frac{d t}{d \tau}\right)=0, \\
-\frac{d}{d \tau}\left(2 g_{r r} \frac{d r}{d \tau}\right)=\frac{\partial g_{t t}}{\partial r}\left(\frac{c d t}{d \tau}\right)^{2}-\frac{\partial g_{r r}}{\partial r}\left(\frac{d r}{d \tau}\right)^{2} .
\end{gathered}
$$

The first integrals of these equations for the unity initial condition and the zero boundary condition at infinity $\left(L=\mathrm{c}^{2}\right)$ can be again easily found:

$$
\begin{gathered}
g_{t t} \frac{d t}{d \tau}=1 \\
g_{t t} g_{r r} \frac{d r}{d \tau} \frac{d r}{d \tau}=c^{2} \eta_{t t}-c^{2} g_{t t}
\end{gathered}
$$

where $\eta_{t t}=1$. The forces acting on the falling test body causing acceleration, similar to Newton's inertial and gravitational forces, are then obtained by differentiating Equation 22 with respect to $\tau$ and dividing the result by $g_{t}$, or directly from EL equations of motion, with the result:

$$
g_{r r} \frac{d^{2} r}{d \tau^{2}}=-\left(\frac{c^{2}}{2} \frac{1}{g_{t t}} \frac{\partial g_{t t}}{\partial \varphi_{n}}\right)_{s} \frac{\partial \varphi_{n}}{\partial r}-\left(g_{r r} \frac{d r}{d \tau} \frac{d r}{d \tau}\right)_{s}\left(\frac{\partial \ln \sqrt{g_{t t} g_{r r}}}{\partial \varphi_{n}}\right)_{s} \frac{\partial \varphi_{n}}{\partial r}
$$

where the metric coefficients are considered as functions of the Newton gravitational potential. In order to keep the number of covariant quantities in the products on both sides of the equation equal, the terms in the parenthesis must be related to scalar quantities as is indicated by the subscript $s$. The acceleration must also be independent of velocity, as is well known. This is satisfied for the spherical coordinates by the relation:

$$
g_{t t} g_{r r}=1
$$

which also follows from the consideration that the space deformation around the centrally gravitating body is locally isotropic. This means that the deformation in the radial direction and the deformation in any angular direction are the same. The space deformation is, of course, detected in the test body trajectory and the isotropic condition can be expressed as follows:

$$
\frac{d \rho}{d r}=\sqrt{g_{r r}}=\frac{\rho}{\sqrt{g_{\varphi \varphi}}}
$$


By substituting into Equation 25 for $g_{\varphi \varphi}$ from Equation 16 the relation in Equation 24, also well known from the Schwarzschild metric, is obtained. Applying this result to Equation 23 then simplifies the acceleration-force equation to read:

$$
g_{r r} \frac{d^{2} r}{d \tau^{2}}=-\left(\frac{c^{2}}{2} \frac{1}{g_{t t}} \frac{\partial g_{t t}}{\partial \varphi_{n}}\right)_{s} \frac{\partial \varphi_{n}}{\partial r}
$$

The same result can also be derived from the consideration that the test body in a free fall follows a geodesic trajectory as is derived in the Appendix. Finally again, to maintain the count of the covariant components equal on both sides of Equation 23 and Equation 26 it is obvious that the metric coefficient for the time coordinate must satisfy the following relation:

$$
\frac{c^{2}}{2} \frac{1}{g_{t t}} \frac{\partial g_{t t}}{\partial \varphi_{n}}=1
$$

The solution of this equation provides the desired relation between the metric coefficient and the potential and together with Equation 4 the final result for the natural coordinate radius:

$$
\begin{gathered}
g_{t t}=e^{2 \varphi_{n} / c^{2}}=e^{-R_{s} / \rho} \\
r=\int_{0}^{\rho} e^{\frac{-R_{s}}{2 \rho}} d \rho
\end{gathered}
$$

From this finding it is then concluded that the gravity is compressing the natural coordinate radius as is shown in Figure 1, but without creating any event horizon. The gravitational field is, therefore, the deformation of the material space entity. Similarly it is for time; the time interval increment measured by a stationary clock in a gravitational field is: $d t=\exp \left(R_{s} / 2 \rho\right) d \tau$ as is well known, Okun' at al. (1999). The gravity is thus clearly deforming the natural space-time.

At this point it is also interesting to compare the gravitational potential resulting from the Schwarzschild metric with the gravitational potential derived above by minimizing the field energy. Using Equation 22 and Equation 24 the acceleration-force equation for a test body falling in the radial direction in the space-time of the Schwarzschild metric is:

$$
\frac{d^{2} r}{d \tau^{2}}=-\frac{c^{2}}{2} \frac{R_{s}}{r^{2}}=-\left(1-\frac{R_{s}}{r}\right) \frac{c^{2}}{2} \frac{R_{s}}{r^{2}-r R_{s}}
$$

The Schwarzschild metric coefficient $g_{t t}$ had to be factored out from the expression to show that the contravariant quantities are equal on both sides of the equation. The Schwarzschild gravitational potential then becomes equal to:

$$
\varphi_{s c h}=\frac{c^{2}}{2} \ln \left(1-\frac{R_{s}}{r}\right) \approx-\frac{\kappa M}{r}+\frac{\kappa^{2} M^{2}}{c^{2} r^{2}}-\ldots
$$

It is now clear that this potential cannot result from minimizing the field energy of the gravitational field and that the variational principle introduced in Equation 5 is not reasonable for finding the metric of a particular mass distribution. The general relationship between the gravitational potential and the metric coefficient as derived in Equation 28 has to be, of course, always maintained.

There have been attempts in the past to rectify the problems of Schwarzschild metric, for example Yilmaz (1958, 1992), who has also arrived at the exponential function for the metric coefficients, but was harshly criticized by Misner (1995) and others. The correct solution is the introduction of the physical coordinate radius as defined by Equation 3, which this author has not found published in the available literature. 


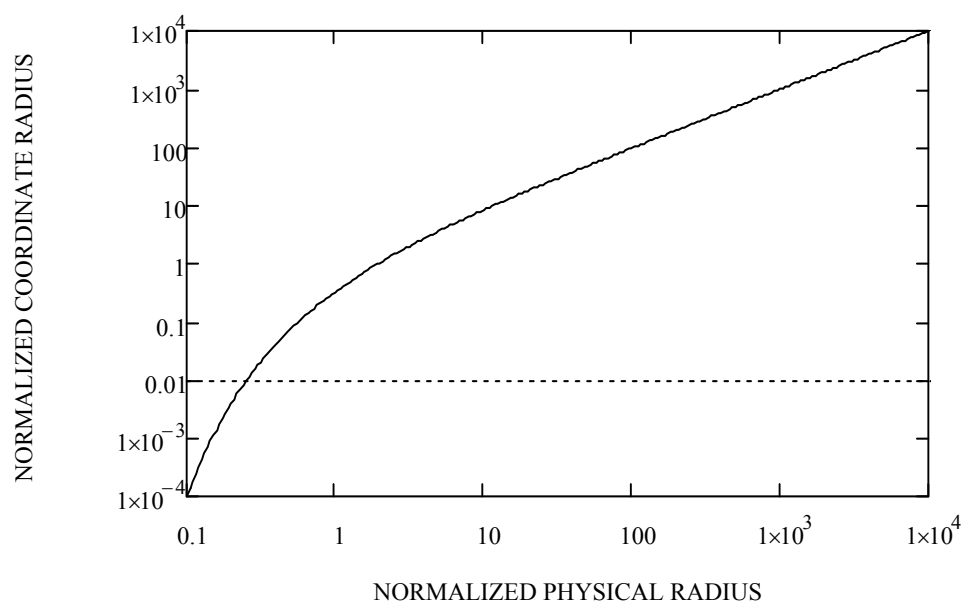

Figure 1. The natural coordinate radius as a function of the physical radius both normalized to $R_{s}$. The dotted line represents the equilibrium state between the field energy and the mass equivalent energy of the body that determines the minimum radius to which any mass, such as a collapsed star, can be compacted to

\section{New Space-time Metric}

Since all the metric coefficients have now been found based on minimizing the gravitational field energy, generating the test body trajectories that conserve the angular momentum and energy, and finally maintaining the contravariance of the acceleration-force equation, the correct metric for the centrally gravitating body is as follows:

$$
d s^{2}=e^{-R_{s} / \rho}(c d t)^{2}-e^{R_{s} / \rho} d r^{2}-\rho^{2} e^{-R_{s} / \rho} d \Omega^{2}, \quad d \rho=e^{R_{s} /(2 \rho)} d r
$$

There are no BHs in the Universe, only very massive and compact gravitating bodies compressed to their minimum possible sizes: $r_{\min } \sim R_{s} / 100$ with no singularity. The light can always escape from them so they can be seen. Particles can also escape from them having enough initial kinetic energy forming, for example, jets of matter, which are often seen emanating from the centers of many galaxies.

If the variational principle in Equation 5 were founded on the sound physical principles then the metrics in Equation 32 and Equation 7 would be identical.

\section{Comparison with Observations}

Having thus obtained the expression for the gravitational potential and, therefore, the expression for the energy of the gravitational field it is possible to find the absolute minimal radius of the centrally gravitating body to which any mass, such as for example a collapsed star, can be compacted to. This is obtained by equating the energy of the field, which is negative, with the mass energy of the compacted body. The total mass-energy of the field plus the body is thus zero:

$$
M c^{2}-\frac{1}{2} \frac{\kappa M^{2}}{\rho_{e q}}=0
$$

In this expression the field energy inside of the massive body was for simplicity neglected, since it is more than an order of magnitude smaller than the mass energy of the body itself. The mass equivalent physical radius then becomes equal to:

$$
\rho_{e q}=\frac{R_{s}}{4}
$$

The corresponding mass equivalent natural coordinate radius is obtained using Equation 29:

$$
r_{e q}=\int_{0}^{\rho_{e q}} e^{\frac{-R_{s}}{2 \rho}} d \rho=\frac{R_{s}}{2} \int_{0}^{1 / 2} e^{\frac{-1}{x}} d x=R_{s} \cdot 0.009384
$$

The radius of the maximally compressed star, for example the star in the center of our galaxy with its immense 
mass of: $M_{G c}=3.0 \cdot 10^{6} M_{S}$, is thus: $r_{\min } \sim R_{s} / 100=83 \cdot 10^{3} \mathrm{~km}$. This is an incredibly small sphere for such a large mass in comparison to our Sun with its radius: $r_{S}=695 \cdot 10^{3} \mathrm{~km}$. It is not the BH since it does not have the event horizon and it is also no singularity. Unfortunately such a small object is very difficult to observe directly, so the false claims of $\mathrm{BH}$ existence will continue for a long time into the future.

The absolute minimum of the gravitational potential that any massive body in the Universe can have on its surface is equal to:

$$
\varphi_{n \min }=-2 c^{2}
$$

This limit also implies that there is a limit to the intrinsic gravitational red shift, which should be verifiable by observations. The maximum intrinsic gravitational red shift observed in the radiation generated when the in-falling matter impacts the surface of the maximally compressed body is thus as follows:

$$
Z_{m x}=e^{2}-1=6.389
$$

The maximum $Z$ shift measured to date is from the Gamma Ray Burst: GRB050904, Cottam at al. (2002) equal to: $Z_{\mathrm{mx}}=6.29$ and from the so called "most distant" Quasar: CFHQS J2329-0301 at $Z=6.43$, both in a reasonable agreement with expectations. Many other Quasars are also exhibiting large intrinsic $Z$ shifts but none of them has so far exceeded the value given in Equation 37.

The most convincing support for the theory, however, comes from the observation of the pulse length of the long duration GRB explosions, when it is considered that these extremely powerful pulses are generated by explosions of the super massive objects located at the centers of galaxies with the estimated mass of approximately equal to: $M_{G}=3.0 \cdot 10^{6} M_{S}$. The pulse duration is obtained by taking the physical mass equivalent radius obtained from Equation 34 and dividing it by the speed of light, which is the maximum possible speed of such an explosion. Finally, this result is corrected for the corresponding maximum time dilation leading to the following simple formula:

$$
\tau_{G R B}=\frac{\kappa M_{s} 3.0 \cdot 10^{6}}{2 c^{3} \sqrt{g_{t t}}}=54.6 \mathrm{sec}
$$

This result compares favorably with the measured data shown in Figure 2 for which there is presently no reasonable explanation. The presence of the second smaller peak in the graph suggests that there should be another population of super massive compact objects in the Universe, possibly Quasars, with the average mass of approximately equal to: $M_{Q}=1.6 \cdot 10^{4} M_{S}$, in addition to the central masses of galaxies. The GRBs from even less massive objects, such as the massive stars or ordinary stars, are not detectable due to the low radiation intensity and the fact that they are not compressed to their minimum possible mass equivalent diameter.

For the Schwarzschild metric, on the other hand, there is no limit to the gravitational potential and the potential can attain values approaching $-\infty$. This implies that any value of the intrinsic gravitational red shift should be observed. However, there is no surface to be impacted, so no radiation can actually be generated by the impacts of the in falling matter. Other concepts of the radiation generation thus must be conjured up with additional adjustable parameters to explain the observations. Again, this is not reasonable and not physical. The infinite gravitational potential at the Schwarzschild radius also precludes any possibility of explosions of BHs and thus the generation of the powerful GRBs. Observations of the substantially larger than the maximum intrinsic gravitational red shifts derived in Equation 37 would, however, falsify the presented theory. 


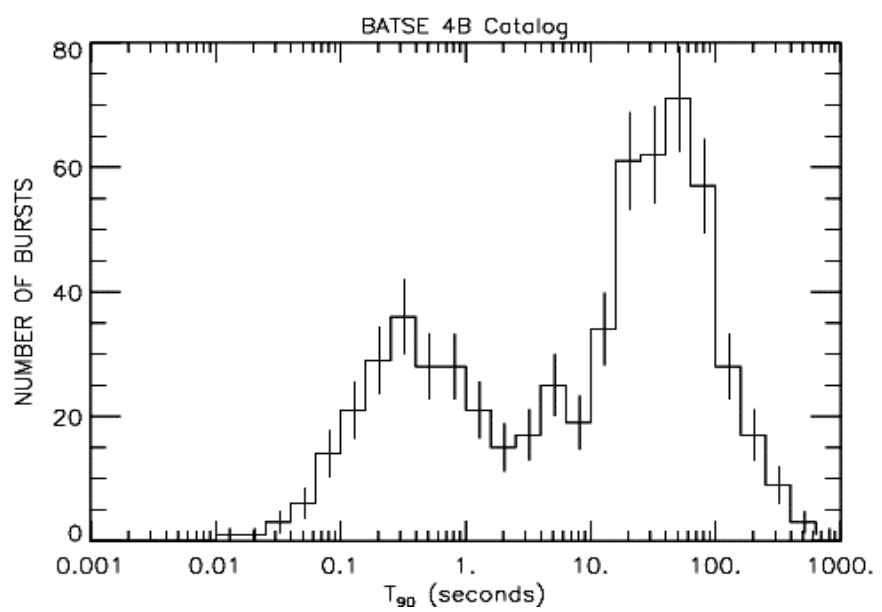

Figure 2. Statistical distribution of the GRB pulse durations as published in the BATSE 4B catalog http://www.batse.msfc.nasa.gov/batse/

\section{Generalization to Arbitrary Mass Configurations}

To generalize the above derived metric for the centrally gravitating body only a small space-time region will be considered where the gravitational field is uniform. The coordinate system for this case can be oriented such that the field is along the $z$ direction. The metric for such space-time is simplified as follows:

$$
d s^{2}=g_{t t}(c d t)^{2}-d x^{2}-d y^{2}-g_{z z} d z^{2}
$$

The metric coefficients along the $x$ and $y$ directions must be unity in order to maintain compatibility with the Lorentz coordinate transformation, which can transform out the gravitational field in the $z$ direction for a free falling coordinate system without any effect on the other two axes. Considering now the test body motion only in the $z$ direction the EL equations of motion for the Lagrangian $\left(L=c^{2}\right)$ corresponding to metric in Equation 39 are:

$$
\begin{gathered}
\left.-\frac{d}{d \tau}\left(2 g_{z z} \frac{d z}{d \tau}\right)=\left(\frac{1}{g_{t t}} \frac{\partial g_{t t}}{\partial \phi_{n}}\right)_{s}\left(g_{t t} \frac{c d t}{d \tau} \frac{c d t}{d \tau}\right)\right)_{s} \frac{\partial \phi_{n}}{\partial z}-\left(\frac{1}{g_{z z}} \frac{\partial g_{z z}}{\partial \phi_{n}}\right)_{s}\left(g_{z z} \frac{d z}{d \tau} \frac{d z}{d \tau}\right)_{s} \frac{\partial \phi_{n}}{\partial z} \\
\frac{d}{d \tau}\left(g_{t t} \frac{d t}{d \tau}\right)=0 .
\end{gathered}
$$

Since the dependency of the metric coefficient $g_{t t}$ on the potential is already known from the previous derivation, it is clear that in order to maintain the covariance of Equation 40 on both sides the metric coefficient $g_{z z}$ must be equal to:

$$
g_{z z}=e^{2 \varphi_{n} / c^{2}}
$$

This simplifies Equation 40 as follows:

$$
\frac{d}{d \tau}\left(g_{z z} \frac{d z}{d \tau}\right)=-\frac{\partial \varphi_{n}}{\partial z}
$$

The metric in Equation 39 then becomes equal to:

$$
d s^{2}=e^{2 \varphi_{n} / c^{2}}(c d t)^{2}-d x^{2}-d y^{2}-e^{2 \varphi_{n} / c^{2}} d z^{2}
$$

In Equation 40 it is, of course, also possible to consider that $g_{z z}=\exp \left(-2 \varphi_{n} / \mathrm{c}^{2}\right)$, however, this choice leads to a disagreement with the Lorentz coordinate transformation and to a variable speed of light in the $z$ direction and thus will not be considered here any further. 
In the next step it will prove useful to evaluate and compare the metric determinants for various space-time metrics. For the metric in Equation 44 it is clear that the following relation holds:

$$
\varphi_{n}=\frac{c^{2}}{2} \ln \sqrt{-g}
$$

Considering that the metric determinant of corresponding physical space-time is $g_{p}=-1$ the relation in Equation 45 can be generalized for any metric and thus for any gravitational field as follows:

$$
\varphi_{n}=\frac{c^{2}}{2} \ln \sqrt{\frac{g}{g_{p}}}
$$

Using Equation 28 to eliminate $\varphi_{n}$ this formula can be further generalized and written as:

$$
g_{t t}^{2}=g / g_{p}
$$

From this result then follows that the gravitational field is compressing the space-time and this is reflected in the corresponding metric determinants. The same relation is also satisfied by the metric derived in Equation 32 . Equation 47 thus imposes a certain deformation restriction on the space-time and thus a condition on the metric coefficients similar to condition of Equation 24. For the Schwarzschild metric, however, the metric determinant is unaffected by the gravity and the gravitational potential using the formula in Equation 46 is:

$$
\varphi_{\text {sch }}=0
$$

This is a contradiction with the result obtained in Equation 31, which again points to a fatal flaw of the Schwarzschild metric and the variational principle of Equation 5. It seems unreasonable that the metric determinant would not be affected by the gravitational field.

The general procedure for finding the metric for any static mass configuration is then as follows: First solving the Poisson equation for the given mass configuration $\rho_{m}$ to find the Newton gravitational potential in the physical space:

$$
\Delta \varphi_{n}=\rho_{m}\left(x_{(p)}^{i}\right)
$$

This step is followed by finding the metric using Equation 47 in a suitable coordinate system with the coordinate time metric coefficient equal to: $g_{t t}=\exp \left(-2 \varphi_{n} / \mathrm{c}^{2}\right)$, and with the known relations between the coordinate and physical distances taking advantage of the mass configuration symmetry and conservation laws of test body trajectories Hynecek (2009b). For the example of uniform gravity field discussed above this is: $\varphi_{n}=g_{0} z_{p}$, where $g_{0}$ is the gravitational field vector pointing in the negative $z$ direction and $z_{p}<0$ is the physical distance. The metric coefficients and the metric line element are then found by solving Equation $42: d z_{p}=\exp \left(g_{0} z_{p} / \mathrm{c}^{2}\right) d z$ for the zero initial condition at $z_{p}=0$ and valid for $z<0$ with the result:

$$
d s^{2}=\left(1-g_{0} z / c^{2}\right)^{-2}(c d t)^{2}-d x^{2}-d y^{2}-\left(1-g_{0} z / c^{2}\right)^{-2} d z^{2}
$$

\section{Agreement with the Lorentz Coordinate Transformation}

The metric for the uniform gravitational field has an advantage that its correctness and thus the correctness of the above approach to the theory of the static gravitational field can be easily verified. The effect of the uniform gravitational field should be simply transformed away in a coordinate system that is free falling in the $z$ direction in such a field. From the first integrals of EL equations of motion corresponding to Lagrangian derived from the metric in Equation 39 it holds that:

$$
g_{t t} \frac{d t}{d \tau}=1, \quad g_{z z}\left(\frac{d z}{d \tau}\right)^{2}=\frac{c^{2}}{g_{t t}}-c^{2}
$$

From this result follows that for a coordinate system falling with the test body it holds:

$$
\left(\frac{d z}{d t}\right)^{2}=c^{2}-c^{2} g_{t t}
$$


The metric coefficients can then be expressed in terms of the $z$ direction velocity as:

$$
g_{t t}=1-v_{z}^{2} / c^{2}
$$

It is important to note that the speed of light in the $z$ direction is constant and equal to $c$, the speed of light in vacuum without any field. The metric in Equation 50 then becomes:

$$
d s^{2}=\left(1-v_{z}^{2} / c^{2}\right)(c d t)^{2}-d x^{2}-d y^{2}-\left(1-v_{z}^{2} / c^{2}\right) d z^{2}
$$

The remaining step in the proof is to use the Lorentz length contraction and the time dilation differential formulas for the system moving in the $z$ direction with a velocity $v_{z}$ :

$$
d t^{\prime}=d t_{z^{\prime}=\text { cnst }} \sqrt{1-v_{z}^{2} / c^{2}}, \quad d x^{\prime}=d x, \quad d y^{\prime}=d y, \quad d z^{\prime}=d z_{t^{\prime}=\text { cnst }} \sqrt{1-v_{z}^{2} / c^{2}}
$$

and obtain the Minkowski flat space-time metric line element of the free falling coordinate system:

$$
d s^{\prime 2}=\left(c d t^{\prime}\right)^{2}-d x^{\prime 2}-d y^{\prime 2}-d z^{\prime 2}
$$

From Equation 53 also follows the famous Einstein's inertial mass-energy equivalence formula. By substituting for the metric coefficient and multiplying the result by the rest mass $m_{0}$ of the test body and by $c^{2}$ the following equation is obtained:

$$
\frac{m_{0} c^{2}}{\sqrt{1-v_{z}^{2} / c^{2}}}=m_{0} c^{2}-m_{0} g_{0} z
$$

The inertial mass-energy of the test body is increased by the energy picked up during the fall in the gravitational field $(\mathrm{z}<0)$. Equation 57 can be easily integrated with the closed form solution written as:

$$
z=c^{2} / g_{0}-\left(c^{2} / g_{0}\right) \sqrt{1+\left(g_{0} t / c\right)^{2}}
$$

This result can then be used to calculate the distance the falling body has travelled as a function of time: For the short times it is: $z \sim-g_{0} t^{2} / 2$, and for the very long times it is: $z \sim-c t$, as can be expected.

It is important to note that the metric in Equation 50 does not satisfy the Einstein field equations, since it does not result in the zero Ricci scalar. The Ricci scalar for this metric was derived by Hynecek (2009b) and it is equal to: $R_{(c)}=2 g_{0}{ }^{2} / c^{4}$. The agreement with the Lorentz coordinate transformation, however, proves the metric correctness and again invalidates the variational principle approach of Equation 5 in finding the correct space-time metric solutions. Since the gravitational field seems to cause the curvature of the space-time it is reasonable to expect that for the uniform gravitational field the Ricci scalar will not be zero.

The fundamental error by assuming that in a vacuum, away from the massive body, the Ricci scalar and consequently the mass energy tensor $T_{j k}$ are zero could not be more apparent than for the case of the uniform gravitational field. This becomes even more obvious when considering that the weak field approximation boundary condition at infinity cannot be applied to this case. The weak field solutions of Einstein field equations are the only solutions that have been confirmed by observations.

\section{Expansion of Metric Coefficients for the Centrally Gravitation Body into a Power Series}

The first order approximation for the metric coefficient $g_{t t}$ and thus for the metric line element of the centrally gravitating non-rotating body are found using Equation 28 and Equation 29 as follows:

$$
r=\int_{0}^{\rho} e^{\frac{-R_{s}}{2 \rho}} d \rho=\frac{R_{s}}{2} \int_{\frac{R_{s}}{2 \rho}}^{\infty} \frac{e^{-x}}{x^{2}} d x=\rho e^{\frac{-R_{s}}{2 \rho}}-\frac{R_{s}}{2} \int_{\frac{R_{s}}{2 \rho}}^{\infty} \frac{e^{-x}}{x} d x=\rho e^{\frac{-R_{s}}{2 \rho}}+\frac{R_{s}}{2} E i\left(-\frac{R_{s}}{2 \rho}\right)
$$

here $\operatorname{Ei}(x)$ is the Euler exponential integral function. Unfortunately there is no analytic expression for $\rho$ as function of $r$, so the approximation needs to be found iteratively. For large distances $(0<x<<1)$, the Euler exponential integral is approximated as:

$$
\operatorname{Ei}(-x)=\ln \left(\gamma^{\prime} x\right)+\ldots
$$

where $\gamma^{\prime}=1.781072 \ldots$ and $\ln \left(\gamma^{\prime}\right)=\gamma$ is the famous Euler constant $\gamma=0.577215 \ldots$. It is therefore possible to write: 


$$
\rho e^{\frac{-R_{s}}{2 \rho}}=r-\frac{R_{s}}{2} \ln \left(\frac{\gamma^{\prime} R_{s}}{2 \rho}\right)+\ldots
$$

Rearranging this result as follows:

$$
\frac{1}{\rho}=\frac{1}{r} e^{\frac{-R_{s}}{2 \rho}}+\frac{R_{s}}{2 r \rho} \ln \left(\frac{\gamma^{\prime} R_{s}}{2 \rho}\right)+\ldots=\frac{1}{r}-\frac{R_{s}}{2 r \rho}+\frac{R_{s}}{2 r \rho} \ln \left(\frac{\gamma^{\prime} R_{s}}{2 \rho}\right)+\ldots
$$

and substituting for $1 / \rho$ from the left hand side of Equation 62 to the right hand side, the iterative expression for $1 / \rho$ valid for large distances becomes:

$$
\frac{1}{\rho}=\frac{1}{r}-\frac{1}{2} \frac{R_{s}}{r^{2}}\left(1+\ln \left(\frac{2 r}{\gamma^{\prime} R_{s}}\right)\right)+\ldots
$$

From this formula then follows the approximation for $g_{t t}$ :

$$
g_{t t}=e^{\frac{-R_{s}}{\rho}}=1-\frac{R_{s}}{r}+\frac{R_{s}^{2}}{r^{2}}\left(1+\ln \sqrt{\frac{2 r}{\gamma^{\prime} R_{s}}}\right)+\ldots
$$

In the next step the second order term in Equation 64 can be neglected since the logarithmic function of $r^{1 / 2}$ increases very slowly and $R_{s}$ is for all practical purposes always very much smaller than $r$. This leads to the familiar formula of the Schwarzschild metric for the metric coefficient $g_{t t}$ :

$$
g_{t t}=1-R_{s} / r+\ldots
$$

Substituting this approximation into Equation 10, and considering that the logarithmic term multiplied by $R_{s} / 2$ in Equation 61 can also be for large distances neglected in comparison to $r$, the formula for the metric line element becomes the celebrated Schwarzschild metric:

$$
d s^{2}=\left(1-R_{s} / r\right) c^{2} d t^{2}-\left(1-R_{s} / r\right)^{-1} d r^{2}-r^{2} d \Omega^{2}
$$

The derived approximation also allows to find a condition that needs to be satisfied to obtain an accurate description of the space-time geometry by this metric $\left(r>R_{s}\right)$. The condition is as follows:

$$
r / R_{s}-\ln \sqrt{r / R_{s}}>>1
$$

From the above derivation there could be no doubt that the Schwarzschild metric is only the first order approximation of the metric introduced in Equation 32 and thus only the first order approximation of reality. The reality is being defined here as a space-time described by a metric that satisfies the contravariance requirement of the test body acceleration-force equation, generates trajectories that conserve the angular momentum, has minimum field energy, and of course also satisfies the four tests of GRT as shown by Hynecek (2007). Since the Schwarzschild metric has been confirmed by observations only for the weak gravitational fields and since it is the first order approximation of the new metric it is, of course, obvious that the new metric also satisfies the weak field tests. The differences will occur only at the strong gravitational fields such as in the vicinity of the neutron stars or the centers of galaxies.

Since the Schwarzschild metric is the correct and unique solution of Einstein field equations for the spherical case, according to the well known Birkhoff theorem as described by Deser (2004), there can be only one inescapable conclusion that the Einstein field equations yield only the first order approximations of the correct metric when the energy-momentum tensor $T_{j k}$ is set to zero.

While the study of Einstein field equations and various Einstein Spaces described by these equations can be an interesting and intellectually rewarding experience with a large amount of work already devoted to this topic by Petrov (1961), it is clear that very little of this work can actually be applied to reality. The Einstein field equations and their various derivatives therefore should not be used to search for the metric to model the strong gravitational fields, or the entire Universe.

\section{Conclusions}

In this paper it was shown that the derivation of Einstein field equations from the variation principle by 
minimizing the Ricci scalar Lagrangian is not the sound physical concept. There is no proof that the Ricci scalar is directly and linearly connected to the minimum of the field energy and thus the resulting field equations do not correspond to reality. This is reflected in the Schwarzschild solution of these equations, which exhibit several problems such as the $\mathrm{BH}$ artifacts and the infinite gravitational potential. The test particle trajectories derived from the Schwarzschild metric also do not conserve the angular momentum and violate the contravariance principle.

The new metric that replaces the Schwarzschild metric has been derived based on the well tested fundamental principles of physics and does not exhibit the above mentioned problems. The metric has as its first order weak field approximation the Schwarzschild metric, so it also satisfies the well know tests of GRT. The new metric, however, does not have the event horizon, which allows finding the minimum possible gravitational potential and the minimum possible size of the gravitating body to which any mass can be compacted to instead of the non-physical singularity of the Schwarzschild metric. Several observations in support of the theory were also presented and discussed. Finally the described principle of metric derivation for the centrally gravitating body was extended to any mass configuration with an example of the metric derivation for the uniform gravitational field.

\section{References}

Cottam, J., Paerels, F., \& Mendez, M. (2002). Gravitationally redshifted absorption lines in the X-ray burst spectra of a neutron star. Nature, 420, 51-54. http://dx.doi.org/10.1038/nature01159

Deser, S. (2004). Schwarzschild and Birkhoff a la Weyl.Retrieved from arXiv:gr-qc/0408067v2

Hynecek, J. (2007). New Space-Time Metric, Four Tests of Gravitation Theory, and Newton's Law of Gravitation. Physics Essays, 20(2), 313. http://dx.doi.org/10.4006/1.3119432

Hynecek, J. (2009a). Retrieved from www.wbabin.net/Science-Journals/Research\%20Papers-Relativity\%20 Theory/Download/1503

Hynecek, J. (2009b). New Space-time Metrics for Symmetric Spaces. Physics Essays, $22(2), 179$. http://dx.doi.org/10.4006/1.3124462

Hynecek, J. (2011). Geometry based critique of general relativity theory. Physics Essays, $24(2), 182$. http://dx.doi.org/10.4006/1.3562500

Logunov, A. A. (1983). Gravitation and Elementary Particle Physics. Moscow: Mir Publishers.

Misner, C. W. (1995). Yilmaz cancels Newton. Retrieved from arXiv:gr-qc/9504050 v2 28 Apr 95.

Okun', L. B., Selivanov, K. G., \& Telegdi, V. L. (1999). Gravitation, photon, clocks. Physics-Uspekhi, 42(10), 1045.

Petrov, A. Z. (1961) Einstein spaces. Fizmatgiz, Moscow 1961; Eng. transl., Oxford: Pergamon Press. http://www.batse.msfc.nasa.gov/batse/

Yilmaz, H. (1958). New approach to General Relativity. Phys. Rev., 111, 1417-1426. http://dx.doi.org/10.1103/PhysRev.111.1417

Yilmaz, H. (1991). Toward a field theory of gravitation. Nuovo Cimento B, 107(8), 941-960. http://dx.doi.org/10.1007/BF02899296

\section{Appendix}

As is well known a free falling body in a gravitational field follows a geodesic trajectory. As a consequence of this fact the covariant derivative of the parameterized (different than coordinate) velocity is zero. The result is equation for the geodesic:

$$
\frac{d^{2} x^{i}}{d \tau^{2}}+\Gamma_{j k}^{i} \frac{d x^{j}}{d \tau} \frac{d x^{k}}{d \tau}=0
$$

where the Christoffel symbols expressed in terms of metric coefficients are equal to:

$$
\Gamma_{j k}^{i}=\frac{1}{2} g^{i m}\left(\frac{\partial g_{m j}}{\partial x^{k}}+\frac{\partial g_{m k}}{\partial x^{j}}-\frac{\partial g_{j k}}{\partial x^{m}}\right)
$$

Considering that the test body falls only in the radial direction, also considering only the radial coordinate and the spherical symmetry, Equation A1 simplifies as follows: 


$$
\frac{d^{2} r}{d \tau^{2}}=g^{r r} \frac{1}{2}\left(-\frac{\partial g_{r r}}{\partial r}\left(\frac{d r}{d \tau}\right)^{2}-\frac{\partial g_{t t}}{\partial r}\left(\frac{c d t}{d \tau}\right)^{2}\right)
$$

This result can be further simplified by using the first integrals from Equation 21 and Equation 22. This leads to the following:

$$
\frac{d^{2} r}{d \tau^{2}}=g^{r r} \frac{c^{2}}{2}\left(-\frac{\partial g_{r r}}{\partial r}\left(1-g_{t t}\right)-\frac{\partial g_{t t}}{\partial r} \frac{1}{g_{t t}^{2}}\right)
$$

Rearranging the right hand side of Equation A4 using the relation: $g_{r r} g_{t t}=1$, the following result is obtained:

$$
\frac{d^{2} r}{d \tau^{2}}=-g^{r r} \frac{c^{2}}{2} \frac{1}{g_{t t}} \frac{\partial g_{t t}}{\partial r}
$$

Since the right hand side of Equation A5 can be considered as being a force term derived from the gradient of some generalized gravitational potential, which approaches the Newton gravitational potential as $r \rightarrow \infty$, it is possible to write:

$$
\frac{d^{2} r}{d \tau^{2}}=-g^{r r} \frac{\partial \varphi_{n}}{\partial r}
$$

By equating the terms on the right hand side of Equation A5 and Equation A6 the equation for the metric coefficient $g_{t t}$ becomes:

$$
\frac{\partial \varphi_{n}}{\partial r}=\frac{c^{2}}{2} \frac{1}{g_{t t}} \frac{\partial g_{t t}}{\partial r}
$$

Finally, by integrating Equation A7, considering the flat space-time at infinity, the result is:

$$
g_{t t}=e^{2 \varphi_{n} / c^{2}}
$$

This is the same formula as in Equation 28, which the metric coefficient $g_{t t}$ must always satisfy. From the above derivation it is also clear that this is a general expression valid for any centrally gravitating body irrespective of the particular form of the gravitational potential. 